PREHOSPITAL CARE

\title{
Desirable attributes of the ambulance technician, paramedic, and clinical supervisor: findings from a Delphi study
}

T Kilner

Emerg Med J 2004;21:374-378. doi: 10.1136/emj.2003.008243

\begin{abstract}
Objectives: To identify those attributes experts regard as desirable qualities in the ambulance technician, paramedic, and clinical supervisor.

Methods: The Delphi technique was used to gain a consensus view from a panel of experts. The first round of the study asked the experts to list the attributes they believed were desirable for the ambulance technician, the ambulance paramedic, and the clinical supervisor. The first round of the study generated 3403 individual statements that were collapsed into 25 broad categories, which were returned to the experts, who were required to rate each of the attributes along a visual analogue scale in respect of each of the identified occupational groups.

Results: On completion of the second round the data were analysed to demonstrate rank ordering of desirable attributes by occupational group. The level of agreement within each group was determined by analysis using the Kendall coefficient of concordance. This showed high levels of agreement within the technician group but less agreement within the paramedic and clinical supervisor group. All were highly significant $\mathrm{p}<0.0001$.

Conclusions: There was significant agreement among the experts as to the desirable attributes of ambulance staff, many of which do not feature in existing ambulance training curriculums. The findings of this study may therefore be of value in informing future curriculum development and in providing guidance for the selection of staff for each of the occupational groups.
\end{abstract} Correspondence to:
Mr T Kilner, The University
of Birmingham, School of
Health Sciences,
Edgbaston, Birmingham
B15 2T,, UK;
t.m.kilner@bham.ac.uk
Accepted for publication
6 August 2003 $\checkmark$ p he past 30 years have seen a remarkable evolution in the provision of prehospital emergency care for the civilian population. Many of the developments have followed on from landmark changes in the training of ambulance staff. Two of the most important turning points have been the publication in 1966 of the Report from the Working Party on Ambulance Equipment and Training, chaired by E L M Millar. ${ }^{1}$ The Millar Report recommended that ambulance crews should receive thorough and intensive training in first aid, subjects of a medical or nursing nature such as the care of seriously ill medical and surgical patients, and nonmedical training in subjects such as communications, lifting and carrying, action at accidents, and sudden illness and driving. Before this the only qualification required to be an ambulance attendant was a first aid certificate issued by one of the recognised voluntary aid societies. There is evidence to suggest that even the possession of a first aid certificate was not always mandatory as a condition of employment. ${ }^{2}$

The next important change in the training of ambulance staff came in the mid-1980s, when in response to concerns about the diversity in local so called advanced training schemes, the Department of Health asked the Standing Medical Advisory Committee to consider the issues surrounding paramedic training. ${ }^{3}$ This evolved, in conjunction with the National Health Service Training Division into the publication Ambulance Service Paramedic Training ${ }^{4}$ and the introduction of the paramedic into the UK ambulance service.

The content of a training programme for ambulance staff is in no small way responsible for defining the occupational groups within the ambulance service. The curriculum for basic training proposed by Millar is skills orientated, as is its modern manifestation Ambulance Service Basic Training, ${ }^{5}$ which is the curriculum basis of the ambulance technician training. The same is true for the ambulance service paramedic training curriculum, ${ }^{6}$ which places emphasis on technical skills and procedures.

From this it might be concluded that the desirable attributes of the paramedic are embedded within the curriculum and that the training programme will produce people with the desirable attributes for the delivering prehospital emergency care. However, it is entirely possible that the curriculum is defining the attributes of the occupational group rather than the desirable attributes being used to define the curriculum. Evidence is emerging that suggests that some may believe the second to be true. In the year 2000 the Joint Royal Colleges Ambulance Liaison Committee (JRCALC) published a discussion paper putting forward the concept of the practitioner in emergency care. ${ }^{7}$ The paper suggested that the current paramedic training programme was leaving people lacking in key attributes such as clinical judgement and limited in others, such as patient assessment.

The occupation of ambulance technician and ambulance paramedic are well established and are associated with a nationally recognised curriculum, albeit the competencies are heavily biased towards technical psychomotor skills. New occupations have begun to appear within the clinical career structure of the ambulance service. These positions, while having a variety of titles, such as clinical supervisor, team leader, and clinical support officer, all seem to have a broadly common purpose, which is the operational clinical supervision of others, management of others as well as the delivery of clinical care in their own right. Given that the desirable attributes of those in traditional occupations are not clearly defined it is possible that the desirable attributes of those in these new roles will also lack clarity of definition. This is perhaps more probable, given the absence of a nationally recognised training or competencies. This lack of a national 
benchmark for clinical supervisors appears to be history repeating itself, with each trust setting its own agenda, just as the advanced training schemes did. The lack of a national lead on this issue will undoubtedly result in no one really knowing what a clinical supervisor is or what they do.

The aim of this study, therefore, is to identify those attributes experts regard as desirable qualities in ambulance personnel who are predominantly engaged in clinical operational activity rather than organisational management.

\section{METHODS}

\section{Study design}

The study adopts an integrated design generating and using both qualitative and quantitative data. This is achieved by the use of the Delphi technique, where the initial questionnaire gathers qualitative data that are used to inform the second questionnaire, which is used to gather quantitative data using a correlational design.

\section{Delphi technique}

The Delphi technique is a process that aims to elicit consensus. It is, as Reid suggests: "A method for the systematic collection and aggregation of informed judgements from a group of experts on specific questions or issues." ${ }^{\prime 8}$ Mead describes this process as having six distinct phases: selection of the expert panel, formulation of the questions, statement generation, reduction and categorisation, rating, and analysis and iteration.'

\section{Selection of the expert panel}

A panel of experts, being knowledgeable about the demands of contemporary prehospital emergency care, were recruited to the study. Chief executives and medical directors or advisors of all ambulance trusts in England, Scotland, and Wales were invited to participate in the study. Members of the JRCALC and examiners for the Diploma in Immediate Medical Care of the Royal College of Surgeons of Edinburgh were also invited to participate as members of the expert panel. Powell suggests it may be possible to create a professional group that represents individual viewpoints and differing opinions while being seen as like minded by outsiders. ${ }^{10}$

Ninety three people were contacted and invited to join the expert panel. At this stage seven experts declined the invitation to participate in the study. These people received no further communication in respect of the study. A further two formally declined to participate in the first round of the data collection, predominantly because of existing workload: they were however included in the second round of data collection.

\section{Formulation of the questions}

A questionnaire was designed, in which respondents were asked to identify desirable qualities for each of three occupational groupings; the ambulance technician, the ambulance paramedic, and the ambulance service clinical supervisor. The respondents were asked to consider their responses in four distinct categories; attitudes and values, knowledge, intellectual skills, and practical or psychomotor skills, based around the classic domains associated with learning; the cognitive domain concerned with knowledge and intellect, the affective domain concerned with attitudes and values, and the psychomotor domain concerned with motor skills. ${ }^{11}$ The cognitive domain was split into knowledge and intellect, as the author was interested in both content, or what they need to know (knowledge) and processes, or utilisation of knowledge (intellect). For the purposes of clarity an example was cited for each of these categories; the example for attitudes and values was "non-judgemental", knowledge was "normal vital signs for adults and children", intellectual skills was "deductive reasoning", and practical or psychomotor skills "basic life support". If the examples were found to form part of the data they would stand on their own merits during the rating phase of the study where the experts would determine the relative merits of the statements.

While directing experts to list desirable attributes of ambulance staff in four categories, the questions posed remained open ended. The initial use of open ended questions, allowing the respondents freedom to generate ideas, is widely recommended in the literature. ${ }^{10} 1213$

\section{Statement generation}

Of the 93 questionnaires distributed in the first round of the study, $34(36.6 \%)$ were completed and returned. All responses were transcribed to create a single text file that contained all responses to each category (attitudes and values, knowledge, intellectual skills, and psychomotor skills) and each occupational group (technician, paramedic, and clinical supervisor). This single block of text was broken down into a list of discrete sentences or bullet points in a process Burnard describes as "sentence-busting". ${ }^{14}$ This process generated 3403 individual sentences or bullet points each being printed out onto a separate card in preparation for reduction and categorisation.

\section{Reduction and categorisation}

Mead describes reduction and categorisation as a process of collapsing two or more statements that express the same idea into a single category thus reducing the number of statements. ${ }^{9}$ This process, also known as content analysis, is described by Cavanagh as the distillation of words into a smaller number of content related categories where the words or phrases share the same meaning. ${ }^{15}$

The data underwent preliminary sorting where each card containing a single sentence or bullet point was examined and then grouped together with cards containing items with the same or similar words and phrase. This process generated 203 narrow categories. These categories underwent a second round of analysis in which the narrow categories were assigned to clusters of similar theme. From this process the data were condensed into 25 broad categories, illustrated in figure 1 .

In an attempt to address issues of validity and reliability a second researcher categorised a second identical set of data blinded to the narrow and broad categories generated by the author. As Hicks suggests, the second researcher was asked to classify the individual statements under generic headings. ${ }^{16}$ There was found to be $84 \%$ agreement between the researchers.

\section{Rating}

The second round of data collection should involve the experts rating the data 9 as part of the process to determine consensus but it also provides an opportunity to assess the internal validity of the data.

After the analysis of the data from the first round a second questionnaire was generated, consisting of 36 attributes based upon the broad categories. Each broad category was represented within the 36 attribute statements, however it was not possible to describe the category referred to as "personal qualities" in a single statement as it had been with all the other categories. This category was expressed in a number of statements, resulting in the 36 statements representing the 25 categories. The respondents were asked to rate the desirability only and not the academic level of that attribute on an unmarked 


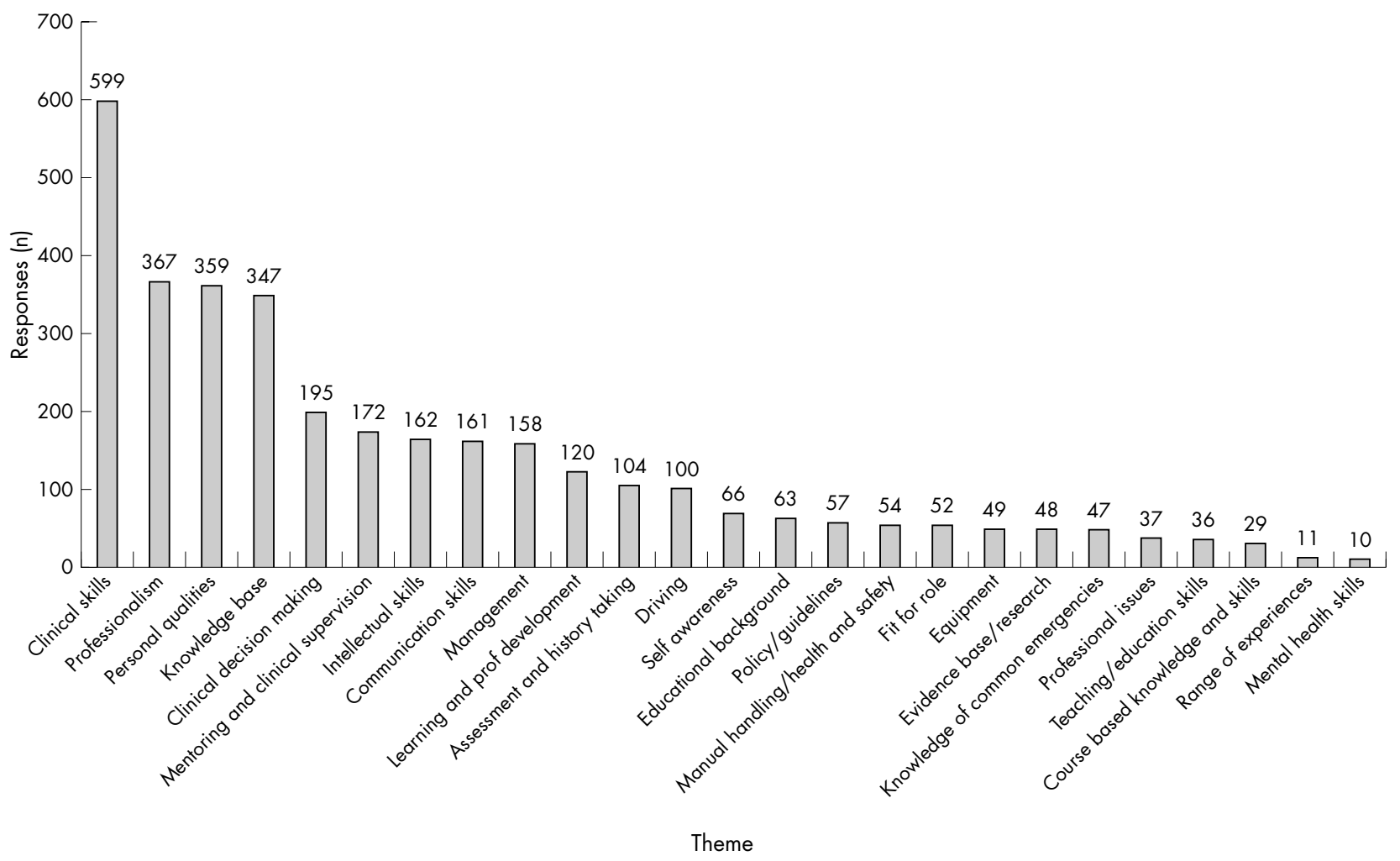

Figure 1 Broad concepts emerging from the first round, identifying the number of individual sentences or bullet points attributed to each concept.

$10 \mathrm{~cm}$ visual analogue scale, from 0 "not at all desirable" to 10 "highly desirable".

\section{Analysis and iteration}

In this study consensus was achieved on completion of the second round. Of the 86 second round questionnaires sent to the expert panel $42(48.8 \%)$ were returned completed. Responses on the visual analogue scores were measured to the nearest $\mathrm{mm}$. The data relating to each of the occupational groups were subject to a Cronbach $\alpha$ coefficient to assess the internal consistency of the scale. An $\alpha$ coefficient of greater than 0.7 is desirable. ${ }^{17}{ }^{18}$ The $\alpha$ coefficient for ambulance technician scales was 0.95, for ambulance paramedic scales 0.96, and for the ambulance clinical supervisor scales 0.95 .

To determine the level of agreement between members of the expert panel the data were analysed using the Kendall coefficient of concordance. ${ }^{16}$ This produces a correlation coefficient (W) between 0 and 1 where 1 represents positive correlation and 0 represents no correlation. This test does not identify negative correlation. Therefore, the closer the score is to 1 the greater the positive correlation. The test also indicates the statistical significance $(p)$ of the correlation coefficient.

\section{RESULTS}

Analysis of the responses relating to the ambulance technician resulted in a correlation coefficient (W) of 0.642 significant at $\mathrm{p}<0.0001$. The correlation coefficient (W) for the ambulance paramedic scales was 0.330 , significant at $\mathrm{p}<0.0001$, and in respect of the ambulance clinical supervisors the correlation coefficient $(\mathrm{W})$ was 0.181 with a significance of $\mathrm{p}<0.0001$.

Cohen suggests that a correlation of 0.5 or above is viewed as a large effect, above 0.3 a medium effect, and 0.1 a small effect. ${ }^{19}$
Attributes, using mean scores, were ranked for each occupational group and are illustrated in table 1.

\section{DISCUSSION}

\section{Levels of agreement}

The literature offers little guidance on the level of agreement required to claim consensus from a Delphi study. Powell ${ }^{10}$ has observed a range of definitions from published studies, including terms such as most participants agree, agreement being implied by the results, or more specifically in numerical terms such as $55 \%$ or $100 \%$.

The Kendall coefficient of concordance relating to the technician scales shows a high level of agreement, or large effect, between the respondents and is highly statistically significant. The level of agreement in relation to the paramedic scales is less than that of the technician, what Cohn regards as a medium effect or good level of agreement, with a high degree of statistical significance. The coefficient for the clinical supervisors indicates a small effect or lower level of agreement that again is highly statistically significant.

Such results are entirely expected in that the role of the technician is well established and that the work required of these people seems to be fairly well understood. A high level of agreement would therefore be anticipated. The role of the paramedic was only developed in recent years and continues to evolve and diversify, while nationally agreed competencies remain at its core. The role of the clinical supervisor is far from clear and is not well established within the ambulance service community at this embryonic stage in its development. Given that the role of the paramedic and clinical supervisor are evolving and maturing, the level of agreement between the respondents will be lower.

\section{Importance of attributes to each occupational group} The rank order of attributes is different for each occupational group, however with four exceptions the mean score for each 
Table 1 Desirability of attributes in rank order for each occupational group based upon mean score

\begin{tabular}{|c|c|c|c|}
\hline Attribute & $\begin{array}{l}\text { Rank (mean score) } \\
\text { Technician }\end{array}$ & Paramedic & Clinical supervisor \\
\hline Honest & $1(93.86)$ & $1(94.17)$ & $1(95.90)$ \\
\hline Patient centred & $2(91.29)$ & $6(91.55)$ & $20(91.74)$ \\
\hline Caring, empathic, and values life & $3(90.63)$ & $5(91.74)$ & $14(93.02)$ \\
\hline Professionalism & $4(89.52)$ & $2(92.80)$ & $4(94.45)$ \\
\hline Non-judgemental, non-discriminatory & $5(88.45)$ & $9(90.52)$ & $3(94.55)$ \\
\hline Self aware, recognising personal responsibilities and limitations & $6(87.93)$ & $12(89.86)$ & $16(92.76)$ \\
\hline Common sense & $7(87.88)$ & $14(87.90)$ & $22(91.64)$ \\
\hline Skills and awareness of manual handling and health and safety & $8(87.86)$ & $17(87.50)$ & $30(86.93)$ \\
\hline Aware of and adherence to national and local guidelines and policies & $9(86.10)$ & $8(90.98)$ & $7(93.95)$ \\
\hline Practical (pragmatic) & $10(86.02)$ & $15(87.86)$ & $24(91.02)$ \\
\hline Familiarity with emergency medical equipment and its operation & $11(85.24)$ & $4(92.31)$ & $10(93.62)$ \\
\hline $\begin{array}{l}\text { Physically fit for the role, manually dextrous with good } \\
\text { hand eye coordination }\end{array}$ & $12(83.52)$ & $23(85.74)$ & $33(80.98)$ \\
\hline Team player with ability to work alone & $13(83.05)$ & $11(89.98)$ & $25(90.88)$ \\
\hline $\begin{array}{l}\text { A range of clinical skills to enable them to manage a range of common } \\
\text { emergency conditions, for example; first aid, life support, } \\
\text { defibrillation, airway management, drug administration }\end{array}$ & $14(81.66)$ & $3(92.40)$ & $9(93.86)$ \\
\hline $\begin{array}{l}\text { Ability to take a history and conduct patient assessment } \\
\text { and examination of both adults and children }\end{array}$ & $15(81.26)$ & $7(91.21)$ & $11(93.55)$ \\
\hline Literate and numerate & $16(80.88)$ & $20(86.67)$ & $23(91.26)$ \\
\hline Skills and underpinning knowledge of advanced driving & $17(80.55)$ & $29(76.36)$ & $35(74.81)$ \\
\hline $\begin{array}{l}\text { Awareness of, and regard for, professional issues such as; autonomy, } \\
\text { accountability, confidentiality and a code of professional conduct }\end{array}$ & $18(79.85)$ & $16(87.52)$ & $15(92.79)$ \\
\hline Communication skills; written, verbal, and electronic & $19(79.50)$ & $18(87.48)$ & $26(90.52)$ \\
\hline Commitment to self development & $20(79.36)$ & $13(88.19)$ & $5(94.26)$ \\
\hline $\begin{array}{l}\text { Ability to learn including the ability to use reflection and } \\
\text { learn from experience }\end{array}$ & $21(77.24)$ & $19(86.88)$ & $19(91.79)$ \\
\hline Familiarity with local operational area & $22(76.52)$ & $28(79.74)$ & $34(76.98)$ \\
\hline $\begin{array}{l}\text { Underpinning knowledge of common emergency conditions } \\
\text { in adults and children }\end{array}$ & $23(75.95)$ & $10(90.12)$ & $6(94.26)$ \\
\hline Mental health skills & $24(70.79)$ & $27(82.26)$ & $32(85.55)$ \\
\hline Role model to others & $25(69.52)$ & $25(84.00)$ & $8(93.90)$ \\
\hline Awareness of evidenced based practice & $26(63.74)$ & $26(82.55)$ & $27(89.98)$ \\
\hline $\begin{array}{l}\text { Underpinning knowledge in biological sciences including } \\
\text { anatomy, physiology, and pathology }\end{array}$ & $27(60.31)$ & $24(85.05)$ & $13(93.21)$ \\
\hline $\begin{array}{l}\text { Intellectual skills to enable the interpretation of clinical data, } \\
\text { the implementation of clinical judgement and decision making, } \\
\text { and the formulation of a diagnosis }\end{array}$ & $28(60.21)$ & $21(86.45)$ & $17(92.19)$ \\
\hline Underpinning knowledge in law and ethics & $29(59.33)$ & $30(78.71)$ & $28(89.50)$ \\
\hline $\begin{array}{l}\text { Skills and knowledge from recognised emergency care courses } \\
\text { such as; Pre Hospital Trauma Life Support (PHTLS), Advanced Life } \\
\text { Support (ALS), Pre Hospital Paediatric Life Support (PHPLS) }\end{array}$ & $30(58.98)$ & $22(86.45)$ & $18(91.98)$ \\
\hline $\begin{array}{l}\text { An understanding of current issues, clinical governance, and the } \\
\text { role of others within the wider health economy }\end{array}$ & $31(57.45)$ & $33(75.71)$ & $21(91.71)$ \\
\hline Skills in clinical and personnel management & $32(54.98)$ & $32(75.93)$ & $12(93.43)$ \\
\hline Brave & $33(47.86)$ & $36(51.64)$ & $36(54.74)$ \\
\hline Leadership, mentoring, and supervision skills & $34(46.98)$ & $31(76.81)$ & $2(95.62)$ \\
\hline Research, statistics, and information retrieval skills & $35(42.60)$ & $34(73.81)$ & $29(88.90)$ \\
\hline Skills in teaching and presentation & $36(38.29)$ & $35(59.83)$ & $31(86.52)$ \\
\hline
\end{tabular}

attribute is greater for the paramedic than the technician and greater for the clinical supervisor than the paramedic. It seems therefore that a wider range of attributes is desired of the clinical supervisor than the paramedic and technician and that while the range of desirable attributes of the paramedic is less than the clinical supervisor it is more extensive than that of the technician. The exceptions to this are "skills and awareness in manual handling and health and safety" and "skills and underpinning knowledge of advanced driving" where these attributes are seen as having greater desirability in the technician than the paramedic and greater desirability in the paramedic than the clinical supervisor. Two of the attributes, "physically fit for the role, manually dextrous with good hand eye coordination" and "familiarity with the local operational area" are seen as being more desirable in the paramedic than in either the clinical supervisor or the technician.

The scores for health and safety and manual handling are broadly similar, which would suggest that these are issues of equal importance across the occupational groups. The suggestion that driving skills are more important for the technician than the paramedic or clinical supervisor may hint at a distinction between driver and attendant, a distinction that appeared in the ambulance service of the past and that currently is seen in ambulance services around the world. This is however by no means conclusive. It is unclear why it would be desirable that the paramedic is physically fitter, more manually dextrous, and with better hand eye coordination than either the technician or the clinical supervisor or why they should be more familiar with the operational area.

\section{Reflection of desirable attributes in curriculum}

The technician and paramedic curriculum ${ }^{20}$ has a technical skills based focus and makes little acknowledgement of attributes beyond this, which commonly appear within the curriculums of other healthcare provides, and have been identified by the expert group. In this study clinical skills and the policies and guidelines supporting them receive high mean scores for all the occupational groups. However, issues such as self awareness, culture, and ethnicity, evidenced based practice, research, mentoring and supervision, clinical governance, teaching, and presentation skills, for example, also receive comparatively high mean scores yet do not explicitly feature within the curriculum. Other attributes that 
do appear in the curriculum lack the detail to attain the extent the expert panel suggest as being desirable, for example the curriculum, while including elements concerning the interpretation of clinical data, does not prepare the student to exercise clinical judgement to any great extent nor does it enable them to formulate a diagnosis. Many of the highly rated attributes desired of the clinical supervisor are not featured in their former training as ambulance technician and ambulance paramedic, nor are they part of a nationally agreed benchmark of competencies. It therefore seems possible that development of personnel for these roles will be suboptimal if the desirable attributes have not been articulated and mapped into a professional development package.

It is possible that where the technician or paramedic curriculum is embedded within a diploma or degree programme some or all of these desirable attributes may be addressed. It is however true that the vast majority of technicians and paramedics trained in the United Kingdom are trained through a traditional curriculum and not via a diploma or degree programme.

\section{New roles}

New roles continue to be developed within the ambulance service, of these roles the practitioner in emergency care is receiving much attention. There is an absence of published literature that clearly defines the role these personnel will undertake, the contribution they will make to the delivery of prehospital care, and the attributes required of these people to deliver on those aspirations. It would appear there is an appetite to develop a curriculum or curriculums to educate these people without knowing what attributes with which they should exit from training programme. The danger of this is that the practitioner will again be defined by the curriculum rather than the curriculum being designed to meet the needs of the practitioner.

\section{Personal qualities}

Personal qualities are ranked highly in all occupational groups and honesty being ranked at number 1 in all three groups. While a curriculum may include issues relating to culture and ethnicity for example, these qualities should perhaps be considered when recruiting candidates to service, and during progress selection.

\section{Conclusion}

The ambulance service has made rapid progress in its comparatively short history, no more so than in its training of those delivering the care to patients. It does however seem that the curriculum has become the driving force for defining the occupational group rather than the desirable attributes of the professional group defining the curriculum. This study has endeavoured to identify some of those desirable attributes in three occupational groups, seen within contemporary ambulance service practice in the UK. Many of these desirable attributes do not feature in current training curriculums for operational ambulance staff. This study may offer some guidance on the future direction of technician and paramedic training and the professional development of the clinical supervisor. It may further offer guidance in the selection of clinical staff at the point of entry to the service and at the point of progression. While not offering guidance on the content of a curriculum for the practitioner in emergency care, it affords a reminder that there is a danger that once again the practitioner may become defined by the curriculum.

\section{ACKNOWLEDGEMENTS}

Thanks to Professor Carolyn Hicks, for commenting on the design of the study, the data collection tools, and the draft manuscript, in addition to the valuable statistical advice. Thanks also to Maggie Griffiths, Research Fellow for undertaking the independent categorisation of the first round data.

Conflicts of interest: TK is an examiner for the Diploma in Immediate Medical Care, Royal College of Surgeons of Edinburgh and a member of the JRCALC. This project was undertaken as part of work contributing to his PhD study.

\section{REFERENCES}

1 Ministry of Health, Scottish Home and Health Department. Report by the working party on ambulance training and equipment: part 1-training. London: HMSO, 1966.

2 Spears R. Patients are people: an account of the Salford Ambulance Service 1948-1974. Salford: Neil Richardson, 1994.

3 Caple L. A concise history of ambulance services in Great Britain. Ambulance UK 2001;16:295-7.

4 NHSTD. Ambulance service paramedic training. Bristol: The National Health Service Training Division, 1991.

5 IHCD. Ambulance service basic training. Bristol: Institute of Health and Care Development, 1998.

6 IHCD. Ambulance service paramedic training. Bristol: IHCD Health and Care Ltd, 1999.

7 The Joint Royal Colleges and Ambulance Liaison Committee and The Ambulance Service Association. The future role and education of paramedic ambulance personnel (emerging concepts). London: JCALC/ASA, 2000.

8 Reid N. The Delphi technique: its contribution to the evaluation of professional practice. In: Ellis R, ed. Professional competence and quality assurance in the caring professions. London: Chapman and Hall, 1989.

9 Mead D, Moseley L. The use of the Delphi as a research approach. Nurse Researcher $2001 ; 8: 4-23$.

10 Powell C. The Delphi technique: myths and realities. Journal of Advanced Nursing 2003;41:376-82.

11 Quinn F. The principles and practice of nurse education. Cheltenham: Stanley Thornes, 2000

12 Hasson F, Keeney S, McKenna H. Research guidelines for the Delphi survey technique. Journal of Advanced Nursing 2003;32:1008-15.

13 Keeney S, Hasson F, McKenna H. A critical review of the Delphi technique as a research methodology for nursing. International Journal of Nursing Studies 2001;38:195-200

14 Burnard P. Analysing data using a word-processor. Nurse Researcher 1994;1:33-42.

15 Cavanagh S. Content analysis: concepts, methods and applications. Nurse Researcher 1997;4:5-16.

16 Hicks C. Research methods for clinical therapists: applied project design and analysis. Edinburgh: Churchill Livingstone, 1999.

17 Pallant J. SPSS survival manual. Buckingham: Open University Press, 2001.

18 Polit D, Beck C, Hungler B. Essentials of nursing research: methods appraisal and utilisation. Philadelphia: Lippincott, 2001.

19 Cohen J, Cohen P. Applied multiple regression correlation analysis for the behavioral sciences. New York: Lawrence Erlbaum, 1975.

20 Ambulance Service Association. IHCD ambulance personnel awards: Training and common core syllabus for ambulance personnel. London: Ambulance Service Association, 2003. 